\title{
Genome-Wide Association Study of Orthostatic Hypotension and Supine-Standing Blood Pressure Changes in Two Korean Populations
}

\author{
Kyung-Won Hong, Sung Soo Kim, Yeonjung Kim* \\ Division of Epidemiology and Health Index, Center for Genome Science, Korea National Institute of Health, \\ Korea Centers for Disease Control and Prevention, Cheongwon 363-951, Korea
}

\begin{abstract}
Orthostatic hypotension $(\mathrm{OH})$ is defined by a $20-\mathrm{mm} \mathrm{Hg}$ difference of systolic blood pressure (dtSBP) and/or a 10-mm Hg difference of diastolic blood pressure (dtDBP) between supine and standing, and $\mathrm{OH}$ is associated with a failure of the cardiovascular reflex to maintain blood pressure on standing from a supine position. To understand the underlying genetic factors for $\mathrm{OH}$ traits $(\mathrm{OH}, \mathrm{dtSBP}$, and dtDBP), genome-wide association studies (GWASs) using 333,651 single nucleotide polymorphisms (SNPs) were conducted separately for two population-based cohorts, Ansung $(n=3,173)$ and Ansan $(n=$ 3,255). We identified 8 SNPs ( 5 SNPs for dtSBP and 3 SNPs for dtDBP) that were repeatedly associated in both the Ansung and Ansan cohorts and had $p$-values of $<1 \times 10^{-5}$ in the meta-analysis. Unfortunately, the SNPs of the OH case control GWAS did not pass our p-value criteria. Four of 8 SNPs were located in the intergenic region of chromosome 2 , and the nearest gene (CTNNA2) was located at $1 \mathrm{Mb}$ of distance. CTNNA2 is a linker between cadherin adhesion receptors and the actin cytoskeleton and is essential for stabilizing dendritic spines in rodent hippocampal neurons. Although there is no report about the function in blood pressure regulation, hippocampal neurons interact primarily with the autonomic nervous system and might be related to $\mathrm{OH}$. The remaining SNPs, rs7098785 of dtSBP trait and rs6892553, rs16887217, and rs4959677 of dtDBP trait were located in the PIK3AP1 intron, ACTBL2-3' flanking, STAR intron, and intergenic region, respectively, but there was no clear functional link to blood pressure regulation.
\end{abstract}

Keywords: genome-wide association study, human CTNNA2 protein, orthostatic hypotension, single nucleotide polymorphism

\section{Introduction}

Orthostasis causes a gravitational shift in circulating blood from the intra-thoracic space to lower extremities and reduces venous return to the heart. This transient increase in sympathetic tone and decrease in parasympathetic tone and these normal cardiovascular reflexes cause an increase in heart rate and vascular resistance, restoring normal blood pressure (BP) and cardiac output [1]. Orthostatic hypotension $(\mathrm{OH})$ is the failure of the cardiovascular reflexes to maintain BP on standing from a supine position [2]. The Malmö Preventive Project reported that individuals with $\mathrm{OH}$ had significantly increased all-cause mortality (hazard ratio, 1.4 to 1.6$)$ [3].

$\mathrm{OH}$ has been defined by the international consensus as a decrease in systolic $\mathrm{BP} \geq 20 \mathrm{~mm} \mathrm{Hg}$ and/or a decrease in diastolic BP $\geq 10 \mathrm{~mm} \mathrm{Hg}$ within 3 mintues of standing [4]. The prevalence of $\mathrm{OH}$ varies from $5 \%$ to $30 \%$, depending greatly on the population being studied [5-7]. Aging is associated with a definite increase in the prevalence of $\mathrm{OH}$ and was reviewed in Benvenuto and Krakoff [2]. In addition to the aging effects, $\mathrm{OH}$ is more prevalent in hypertensive [8, 9], neurological [10], and diabetic patients [11, 12]. The all-cause mortality rate was higher in patients with $\mathrm{OH}$ than in those without $\mathrm{OH}[6,7]$

We have been conducting an epidemiology study of $\mathrm{OH}$ as part of the Korean Genome and Epidemiology Study (KoGES) and previously reported that middle-aged adults (40-70 years), enrolled from 2001 to 2002, had a $12.3 \%$ overall prevalence of $\mathrm{OH}$ and that the $\mathrm{OH}$ frequency in-

Received July 15, 2013; Revised August 2, 2013; Accepted August 6, 2013

*Corresponding author: Tel: +82-43-719-6720, Fax: +82-43-719-6759, E-mail: kimye@korea.kr

Copyright (c) 2013 by the Korea Genome Organization

(c) It is identical to the Creative Commons Attribution Non-Commercial License (http://creativecommons.org/licenses/by-nc/3.0/). 
creased significantly with age, from $6.4 \%$ in those aged 40 44 years to $23.1 \%$ in those aged $65-69$ ( $p<0.001$ ) [9]. In addition, the study confirmed the correlations between $\mathrm{OH}$ and hypertension, body mass index (BMI), and type 2 diabetes [9].

The genetic contribution to $\mathrm{OH}$ can be hypothesized that the frequency of $\mathrm{OH}$ in families with a history of essential hypertension is higher than in the general population [4]. A genetic association study was conducted by Luo et al. [13], and they identified a functional variant of NEDD4L (epithelial sodium channel E3-ubiquitin ligase).

To identify genetic factors associated with $\mathrm{OH}$ trait in Koreans, we conducted genome-wide association studies (GWASs) for $\mathrm{OH}$ case controls using genome-wide single nucleotide polymorphism (SNP) genotypes and also tested supine and standing BPs as quantitative traits.

\section{Methods}

\section{Study subjects}

The study subjects consisted of two population-based cohorts, Ansung and Ansan, which have been conducted as part of the KoGES. The phenotype of the cohort population was previously described [14]. Briefly, the subjects came from the Ansung and Ansan cities, located in Gyeonggi-do province near Seoul, Korea. Written informed consent was obtained from all participants, and this research project was approved by the institutional review board in the Korea National Institute of Health (KNIH).

Subjects with genotype accuracies below 98\% and high missing genotype call rates ( $\geq 4 \%$ ), high heterozygosity $(>30 \%)$, or inconsistency in sex were excluded from subsequent analyses. Individuals who had a tumor were excluded, as were related individuals whose estimated identity-by-state values were high $(>0.80)$. After these quality control steps, 8,842 samples were selected.

\section{Clinical characteristics}

BP was measured 3 times in the supine position. Before the first measurement, participants rested for 5 minutes, and the 3 measurements were taken in one arm showing the higher BP at least 3 minutes apart. After the supine position measurement, the BP was measured soon after standing. Two of 8,842 subjects did not undergo BP measurement in the standing posture. Also, 1,291 individuals who had been treated with anti-hypertensive drug therapy and 1,114 self-reported diabetic patients or diagnosed diabetic patients by KoGES were excluded. Ultimately, 3,255 individuals from Ansan and 3,173 individuals from Ansung were used for the GWASs. Other cardiovascular risk factors, such as cholesterol level and fasting glucose level, were measured from blood samples after overnight fasting.

\section{Study genotypes}

The genotyping of the cohort population was previously described for the Korea Associated Resource (KARE) study [14]. Most DNA samples were isolated from the peripheral blood of participants and genotyped using the Affymetrix Genomewide Human SNP array 5.0 (Affymetrix, Inc., Santa Clara, CA, USA). The quality control steps of genotypes have been described elsewhere [14]. Briefly, the accuracy of the genotyping was determined by Bayesian Robust Linear Modeling using the Mahalanobis Distance (BRLMM) genotyping algorithm [15]. Consequently, 333,651 SNPs had a missing genotype call rate below 0.1, a minor allele frequency (MAF) greater than 0.01, and no deviation from Hardy-Weinberg equilibrium (HWE) $\left(\mathrm{p}>1 \times 10^{-6}\right)$. To examine the population stratification, multidimensional scaling analysis and principal component analysis were performed using 44,724 pruned SNP markers [14].

\section{Statistical analysis}

The orthostatic BP changes were calculated by deltaBP = mean supine position BP - standing position BP. Delta systolic blood pressures (dtSBP), delta diastolic blood pressure (dtDBP), and OH cases (dtSBP $\geq 20$ and/or dtDBP $\geq 10$ [16]) were analyzed by linear or logistic regression, controlling for covariates, such as age, sex, BMI, and systolic BP. Statistical analyses were performed using PLINK version 1.07, using default options [17] and SPSS version 15.0 (SPSS Inc., Chicago, IL, USA). The GWAS were conducted for Ansan and Ansung separately. The Ansan GWAS and Ansung replication study results were combined by inversevariance meta-analysis method, assuming fixed effects, with Cochran's Q test used to assess between-study heterogeneity [18]. The selected SNPs had a common MAF $(>0.05)$ and the population heterogeneity $(p>0.05)$. The asymptotic HWE tests were conducted using PLINK, and all the reported p-values were two-sided.

\section{Results}

\section{Clinical characteristics}

Table 1 describes the clinical characteristics of $\mathrm{OH}$ case and control subjects in the Ansan and Ansung cohorts; 311 participants (7.6\%) and 496 participants (14.3\%) were found to have $\mathrm{OH}$ in Ansan and Ansung, respectively. The gender distributions of $\mathrm{OH}$ differed significantly in both cohorts, and more male $\mathrm{OH}$ in Ansung and less male $\mathrm{OH}$ in Ansan were observed. The age of $\mathrm{OH}$ in Ansung (58.8 \pm 7.8 years) was older than that in Ansan $(51.5 \pm 8.3$ years $)$, reflecting the higher prevalence of $\mathrm{OH}$ in Ansung. 
Table 1. Clinical characteristics of orthostatic hypotension case controls in Ansan and Ansung cohorts phenotype

\begin{tabular}{|c|c|c|c|c|c|c|c|}
\hline \multirow{3}{*}{ Phenotype } & \multicolumn{7}{|c|}{ Orthostatic hypotension } \\
\hline & \multicolumn{3}{|c|}{ Ansan $(n=3,255)$} & \multicolumn{3}{|c|}{ Ansung $(n=3,173)$} & \multirow{2}{*}{$\begin{array}{c}\text { Ansan vs. Ansung } \\
\text { p-value }\end{array}$} \\
\hline & Control & Case & p-value & Control & Case & p-value & \\
\hline n $(\%)$ & $3,015(92.6)$ & $240(7.4)$ & - & $2,746(86.5)$ & 427 (13.5) & - & \\
\hline Male (\%) & $1,517(50.3)$ & $106(44.2)$ & $<0.01$ & $1,114(40.5)$ & $269(63.0)$ & $<0.001$ & $<0.01$ \\
\hline Age $(y)$ & $47.9 \pm 7.4$ & $51.3 \pm 8.3$ & $<0.001$ & $54.8 \pm 8.8$ & $59.8 \pm 7.8$ & $<0.001$ & $<0.001$ \\
\hline Height $(\mathrm{cm})$ & $162.1 \pm 8.4$ & $159.6 \pm 8.0$ & $<0.001$ & $158.6 \pm 8.6$ & $159.2 \pm 8.2$ & 0.1764 & $<0.001$ \\
\hline Body mass index $\left(\mathrm{kg} / \mathrm{m}^{2}\right)$ & $24.6 \pm 2.9$ & $24.4 \pm 3.0$ & 0.1860 & $24.3 \pm 3.2$ & $23.6 \pm 3.3$ & $<0.001$ & 0.1860 \\
\hline Supine SBP (mm Hg) & $110.9 \pm 15.9$ & $119.4 \pm 17.9$ & $<0.001$ & $118.9 \pm 16.8$ & $129.7 \pm 16.5$ & $<0.001$ & $<0.001$ \\
\hline Supine DBP (mm Hg) & $71.8 \pm 11.8$ & $77.1 \pm 11.4$ & $<0.001$ & $75.8 \pm 9.9$ & $81.4 \pm 9.3$ & $<0.001$ & $<0.001$ \\
\hline Standing SBP (mm Hg) & $114.9 \pm 16.6$ & $105.2 \pm 19.9$ & $<0.001$ & $116.9 \pm 18.7$ & $105.0 \pm 16.2$ & $<0.001$ & $<0.001$ \\
\hline Standing DBP (mm Hg) & $77.6 \pm 11.9$ & $64.6 \pm 14.7$ & $<0.001$ & $81.4 \pm 13.0$ & $75.2 \pm 12.0$ & $<0.001$ & $<0.001$ \\
\hline Delta SBP (mm Hg) & $4.0 \pm 10.6$ & $-14.2 \pm 14.3$ & $<0.001$ & $-2.1 \pm 10.4$ & $-24.7 \pm 8.7$ & $<0.001$ & $<0.001$ \\
\hline Delta DBP (mm Hg) & $5.8 \pm 8.4$ & $-12.5 \pm 9.5$ & $<0.001$ & $5.6 \pm 8.8$ & $-6.1 \pm 9.9$ & $<0.001$ & $<0.001$ \\
\hline
\end{tabular}

Values are number $(\%)$ or mean \pm SD.

$\mathrm{SBP}$, systolic blood pressure; DBP, diastolic blood pressure.

(A)

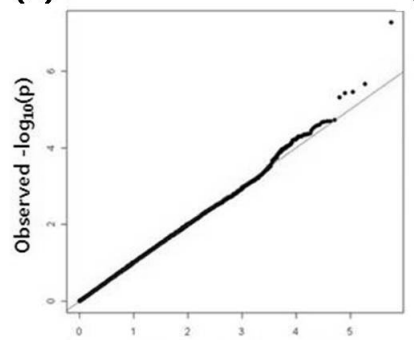

(D)

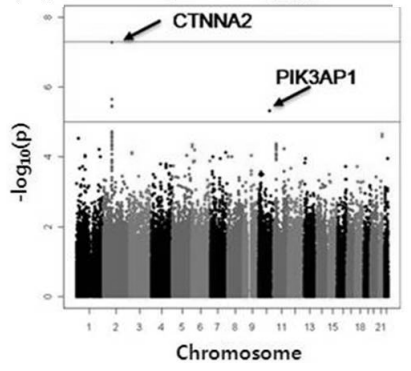

(B)

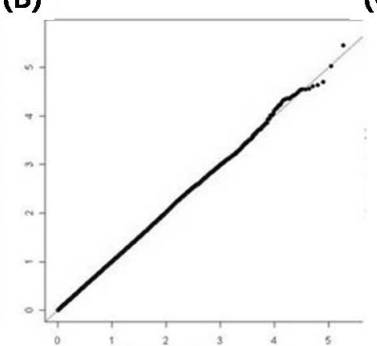

(E)

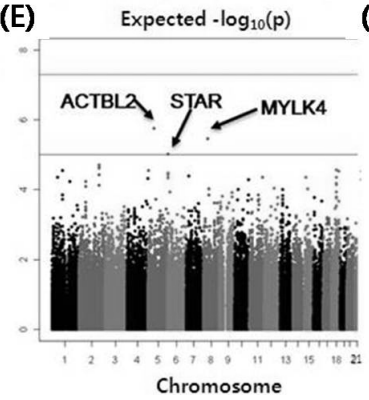

(C)

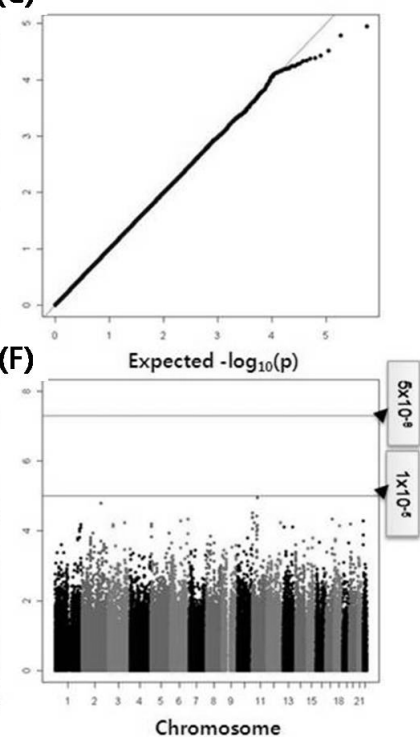

Fig. 1. Quantile-quantile plots and Manhattan plots of genomewide association studies for delta systolic blood pressure $(A, D)$, delta diastolic blood pressure $(B$, $\mathrm{E})$, and orthostatic hypotension (C, F).
The BMI was significantly smaller in Ansung $\mathrm{OH}$ cases than controls, but it was not significant in Ansan. While the mean supine BP was significantly higher in $\mathrm{OH}$ than controls, the standing $\mathrm{BP}$ was significantly lower in $\mathrm{OH}$ than controls.

\section{Genome-wide association studies}

GWAS were conducted for each cohort, and the target phenotypes were dtSBP, dtDBP, and $\mathrm{OH}$. The GWAS results of each cohort were combined by meta-analyses, and quantile-quantile plots and Manhattan plots are depicted in Fig. 1.

All detected SNPs are described in Table 2. Five SNPs for
dtSBP trait and three SNPs for dtDBP trait passed our criteria (replicated in both cohorts [Ansung and Ansan] and $\mathrm{p}<1 \times$ $10^{-5}$ ), but unfortunately, the SNPs of the $\mathrm{OH}$ case control GWAS did not pass our p-value criteria. Among the significant SNPs, four SNPs (rs6736587, rs6756959, rs6715049, and rs17020502) were in the high linkage disequilibrium status $\left(\mathrm{r}^{2}>0.9\right)$ and were including the most significant SNP, rs6736587 (meta-analysis p-value, $5.3 \times 10^{-8}$ ). The region of four SNPs was located in the intergenic region of chromosome 2 , and the nearest gene (CTNNA2) was located at $1 \mathrm{Mb}$ of distance. The remaining SNPs revealed a suggestive association $\left(\mathrm{p}<1 \times 10^{-5}\right)$ with $\mathrm{dtSBP}$ at $\mathrm{rs} 7098785$ (PIK3AP1 intron) and with dtDBP at rs6892553, rs16887217, 
Table 2. Significant SNPs of genomewide association study and replication results

\begin{tabular}{|c|c|c|c|c|c|c|c|c|c|c|c|}
\hline CHR & RSID & BP & $\begin{array}{c}\text { Proxy } \\
\text { gene }\end{array}$ & Function & $\begin{array}{l}\text { Minor } \\
\text { allele }\end{array}$ & MAF & Area & Beta $\pm \mathrm{SE}$ & p-value & Heterogenity & Cochran Q \\
\hline \multicolumn{12}{|c|}{ Delta SBP } \\
\hline \multirow[t]{3}{*}{2} & rs6736587 & 81709236 & CTNNA2 & Intergenic & C & 0.16 & Ansan & $-1.74 \pm 0.33$ & $2.0 \times 10^{-7}$ & & \\
\hline & & & & & & & Ansung & $-0.89 \pm 0.39$ & $2.3 \times 10^{-2}$ & & \\
\hline & & & & & & & Meta-analysis & -1.38 & $5.3 \times 10^{-8}$ & 63.16 & 0.1 \\
\hline \multirow[t]{3}{*}{2} & rs6756959 & 81626234 & CTNNA2 & Intergenic & A & 0.155 & Ansan & $-1.60 \pm 0.34$ & $2.2 \times 10^{-6}$ & & \\
\hline & & & & & & & Ansung & $-0.69 \pm 0.40$ & $8.8 \times 10^{-2}$ & & \\
\hline & & & & & & & Meta-analysis & -1.22 & $2.2 \times 10^{-6}$ & 67.14 & 0.08 \\
\hline \multirow[t]{3}{*}{2} & rs6715049 & 81626505 & CTNNA2 & Intergenic & $\mathrm{C}$ & 0.155 & Ansan & $-1.57 \pm 0.34$ & $3.5 \times 10^{-6}$ & & \\
\hline & & & & & & & Ansung & $-0.67 \pm 0.40$ & $9.3 \times 10^{-2}$ & & \\
\hline & & & & & & & Meta-analysis & -1.20 & $3.5 \times 10^{-6}$ & 65.88 & 0.09 \\
\hline \multirow[t]{3}{*}{2} & rs17020502 & 81681572 & CTNNA2 & Intergenic & A & 0.06 & Ansan & $-1.73 \pm 0.51$ & $6.7 \times 10^{-4}$ & & \\
\hline & & & & & & & Ansung & $-1.93 \pm 0.61$ & $1.7 \times 10^{-3}$ & & \\
\hline & & & & & & & Meta-analysis & -1.81 & $3.7 \times 10^{-6}$ & 0 & 0.8 \\
\hline \multirow[t]{3}{*}{10} & rs7098785 & 98407920 & PIK3AP1 & Intron & $\mathrm{T}$ & 0.361 & Ansan & $-0.76 \pm 0.25$ & $2.7 \times 10^{-3}$ & & \\
\hline & & & & & & & Ansung & $-1.10 \pm 0.31$ & $4.0 \times 10^{-4}$ & & \\
\hline & & & & & & & Meta-analysis & -0.90 & $4.9 \times 10^{-6}$ & 0 & 0.39 \\
\hline \multicolumn{12}{|c|}{ Delta DBP } \\
\hline \multirow[t]{3}{*}{5} & rs6892553 & 56845895 & ACTBL2 & 3' Flanking & G & 0.137 & Ansan & $1.22 \pm 0.30$ & $5.2 \times 10^{-5}$ & & \\
\hline & & & & & & & Ansung & $0.90 \pm 0.34$ & $8.5 \times 10^{-3}$ & & \\
\hline & & & & & & & Meta-analysis & 1.08 & $1.8 \times 10^{-6}$ & 0 & 0.48 \\
\hline \multirow[t]{3}{*}{8} & rs16887217 & 38123582 & STAR & Intron & C & 0.105 & Ansan & $0.95 \pm 0.34$ & $4.7 \times 10^{-3}$ & & \\
\hline & & & & & & & Ansung & $1.42 \pm 0.37$ & $1.5 \times 10^{-4}$ & & \\
\hline & & & & & & & Meta-analysis & 1.16 & $3.5 \times 10^{-6}$ & 0 & 0.35 \\
\hline \multirow[t]{3}{*}{6} & rs4959677 & 2445819 & MYLK4 & Intergenic & $\mathrm{C}$ & 0.216 & Ansan & $0.97 \pm 0.25$ & $1.1 \times 10^{-4}$ & & \\
\hline & & & & & & & Ansung & $0.65 \pm 0.28$ & $2.2 \times 10^{-2}$ & & \\
\hline & & & & & & & Meta-analysis & 0.83 & $9.4 \times 10^{-6}$ & 0 & 0.4 \\
\hline
\end{tabular}

SNP, single nucleotide polymorphism; CHR, chromosome; RSID, reference SNP ID obtained from dbSNP database; BP, base pair based on the human reference genome, ver. $36(\mathrm{NCBI})$; proxy gene, candidate functional gene around $\pm 1 \mathrm{Mbp}$; MAF, minor allele frequency; SBP, systolic blood pressure; DBP, diastolic blood pressure.

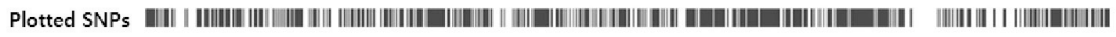

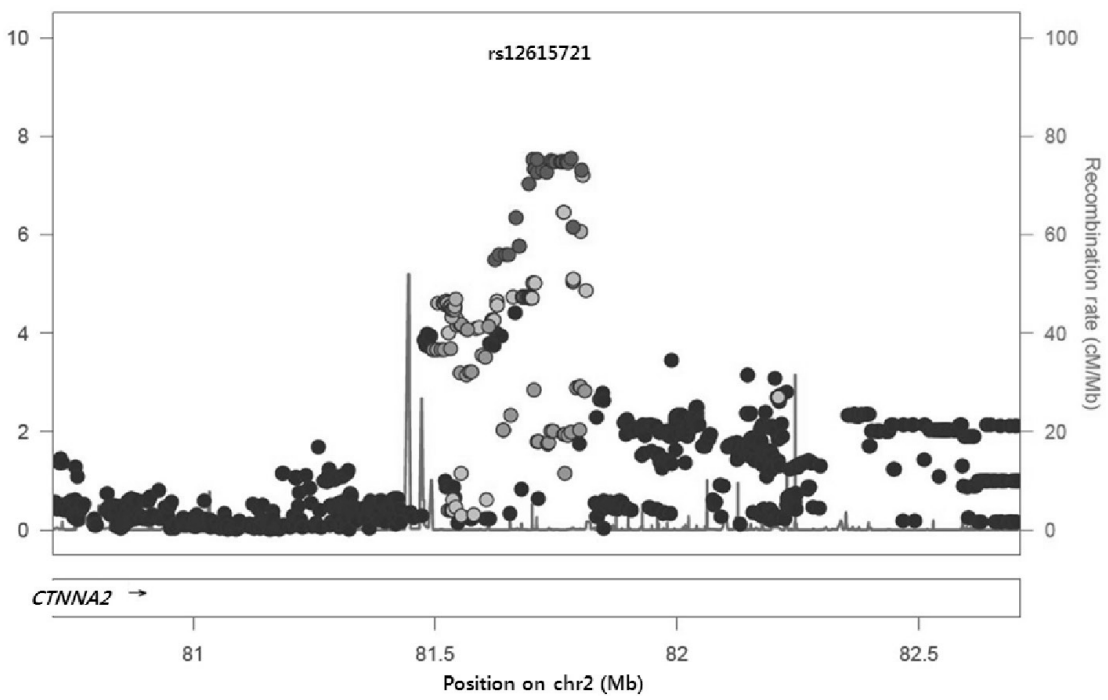

Fig. 2. Signal plot of the most significant association with delta systolic blood pressure. SNP, single nucleotide polymorphism. 
and rs4959677, which were located in the ACTBL2 3' flanking, steroidogenic acute regulatory protein (STAR) intron, and intergenic regions (chromosome 6p 25.2), respectively.

\section{Discussion}

These normal baro-reflex responses cause an increase in heart rate and vascular resistance, restoring normal BP and cardiac output [1]. $\mathrm{OH}$ is the failure of the cardiovascular reflexes to maintain $\mathrm{BP}$ on standing from a supine position [2]. Also, the frequency of $\mathrm{OH}$ in families with a history of essential hypertension may be higher than in the general population [4]. Therefore, the identification of genetic factor for orthostasis will help us understand $\mathrm{OH}$ mechanisms.

The signal plot for the most significant SNP (rs6736587) is depicted in Fig. 2; the region was intergenic and a functional gene was located $1 \mathrm{Mbp}$ upstream. Interestingly, this region is known as the chromosome 2p12-11.2 deletion syndrome region, and several cases report mental or psychomotor retardation $[19,20]$. There was only one gene, CTNNA2 (or known as alpha-N-catenin), around $\pm 1 \mathrm{Mbp}$. CTNNA2 is a linker between cadherin adhesion receptors and the actin cytoskeleton and is essential for stabilizing dendritic spines in rodent hippocampal neurons [21]. Although there is no report about function in BP regulation, hippocampal neurons interact primarily with the autonomic nervous system. Therefore, the CTNNA2 gene may function in orthostatic BP regulation.

Among the other SNPs, rs7098785 was located in the phosphoinositide-3-kinase adaptor protein 1 (PIK3AP1) gene. PIK3AP1 is involved in B-cell development [22]. Interestingly, in silico analyses by the UCSC genome browser (http://genome.ucsc.edu/) indicated that the SNP was located in a DNase I hypersensitive region and in the binding site of transcription factors (MEF2C, BATF, MEF2A). Therefore, the rs7098785 SNP might have a regulatory function of PIK3AP1 expression.

The remaining three SNPs of dtDBP were located in the ACTBL2 (beta-actin like protein 2) 3' flanking region, STAR intron, and intergenic region (chromosome $6 \mathrm{p} \mathrm{25.2).} \mathrm{The}$ ACTBL2 protein is involved in various types of cell motility [23], and STAR is involved in the regulation of steroid hormone synthesis [24].

Our study was the first trial to identify genetic variants for orthostatic BP traits in Koreans. We hope that our finding could help us understand the underlying mechanism of orthostatic BP changes and related diseases.

\section{References}

1. Mathias CJ. Orthostatic hypotension: causes, mechanisms, and influencing factors. Neurology 1995;45(4 Suppl 5):S6-S11.

2. Benvenuto LJ, Krakoff LR. Morbidity and mortality of orthostatic hypotension: implications for management of cardiovascular disease. Am J Hypertens 2011;24:135-144.

3. Fedorowski A, Stavenow L, Hedblad B, Berglund G, Nilsson PM, Melander O. Orthostatic hypotension predicts all-cause mortality and coronary events in middle-aged individuals (The Malmo Preventive Project). Eur Heart J 2010;31:85-91.

4. Fedorowski A, Burri P, Melander O. Orthostatic hypotension in genetically related hypertensive and normotensive individuals. J Hypertens 2009;27:976-982.

5. Rutan GH, Hermanson B, Bild DE, Kittner SJ, LaBaw F, Tell GS. Orthostatic hypotension in older adults. The Cardiovascular Health Study. CHS Collaborative Research Group. Hypertension 1992;19(6 Pt 1):508-519.

6. Rose KM, Eigenbrodt ML, Biga RL, Couper DJ, Light KC, Sharrett AR, et al. Orthostatic hypotension predicts mortality in middle-aged adults: the Atherosclerosis Risk In Communities (ARIC) Study. Circulation 2006;114:630-636.

7. Masaki KH, Schatz IJ, Burchfiel CM, Sharp DS, Chiu D, Foley $\mathrm{D}$, et al. Orthostatic hypotension predicts mortality in elderly men: the Honolulu Heart Program. Circulation 1998;98:22902295.

8. Harris T, Lipsitz LA, Kleinman JC, Cornoni-Huntley J. Postural change in blood pressure associated with age and systolic blood pressure. The National Health and Nutrition Examination Survey II. J Gerontol 1991;46:M159-M163.

9. Shin C, Abbott RD, Lee H, Kim J, Kimm K. Prevalence and correlates of orthostatic hypotension in middle-aged men and women in Korea: the Korean Health and Genome Study. J Hum Hypertens 2004;18:717-723.

10. Senard JM, Brefel-Courbon C, Rascol O, Montastruc JL. Orthostatic hypotension in patients with Parkinson's disease: pathophysiology and management. Drugs Aging 2001;18:495505.

11. Krolewski AS, Warram JH, Cupples A, Gorman CK, Szabo AJ, Christlieb AR. Hypertension, orthostatic hypotension and the microvascular complications of diabetes. J Chronic Dis 1985; 38:319-326.

12. Wu JS, Lu FH, Yang YC, Chang CJ. Postural hypotension and postural dizziness in patients with non-insulin-dependent diabetes. Arch Intern Med 1999;159:1350-1356.

13. Luo F, Wang Y, Wang X, Sun K, Zhou X, Hui R. A functional variant of NEDD4L is associated with hypertension, antihypertensive response, and orthostatic hypotension. Hypertension 2009;54:796-801.

14. Cho YS, Go MJ, Kim YJ, Heo JY, Oh JH, Ban HJ, et al. A large-scale genome-wide association study of Asian populations uncovers genetic factors influencing eight quantitative traits. Nat Genet 2009;41:527-534.

15. Rabbee N, Speed TP. A genotype calling algorithm for affymetrix SNP arrays. Bioinformatics 2006;22:7-12.

16. Consensus statement on the definition of orthostatic hypotension, pure autonomic failure, and multiple system atrophy. 
The Consensus Committee of the American Autonomic Society and the American Academy of Neurology. Neurology 1996;46:1470.

17. Purcell S, Neale B, Todd-Brown K, Thomas L, Ferreira MA, Bender D, et al. PLINK: a tool set for whole-genome association and population-based linkage analyses. Am J Hum Genet 2007;81:559-575.

18. Higgins JP, Thompson SG, Deeks JJ, Altman DG. Measuring inconsistency in meta-analyses. BMJ 2003;327:557-560.

19. Prasher VP, Krishnan VH, Clarke DJ, Maliszewska CT, Corbett JA. Deletion of chromosome 2 (p11-p13): case report and review. J Med Genet 1993;30:604-606.

20. Tzschach A, Graul-Neumann LM, Konrat K, Richter R, Ebert G, Ullmann R, et al. Interstitial deletion 2p11.2-p12: report of a patient with mental retardation and review of the literature.
Am J Med Genet A 2009;149A:242-245.

21. Abe K, Chisaka O, Van Roy F, Takeichi M. Stability of dendritic spines and synaptic contacts is controlled by alpha $\mathrm{N}$-catenin. Nat Neurosci 2004;7:357-363.

22. Okada T, Maeda A, Iwamatsu A, Gotoh K, Kurosaki T. BCAP: the tyrosine kinase substrate that connects $\mathrm{B}$ cell receptor to phosphoinositide 3-kinase activation. Immunity 2000;13:817827.

23. Chang KW, Yang PY, Lai HY, Yeh TS, Chen TC, Yeh CT. Identification of a novel actin isoform in hepatocellular carcinoma. Hepatol Res 2006;36:33-39.

24. Strauss JF 3rd, Kallen CB, Christenson LK, Watari H, Devoto $\mathrm{L}$, Arakane $\mathrm{F}$, et al. The steroidogenic acute regulatory protein (StAR): a window into the complexities of intracellular cholesterol trafficking. Recent Prog Horm Res 1999;54:369-394. 